\title{
Association between Uncinate Fasciculus Integrity and Agoraphobia Symptoms in Female Patients with Panic Disorder
}

\author{
Sung Eun Kim ${ }^{1}$, Minji Bang ${ }^{1}$, Eunsoo Won ${ }^{1}$, Sang-Hyuk Lee ${ }^{1,2}$ \\ Departments of ${ }^{1}$ Psychiatry, ${ }^{2}$ Clinical Pharmacology and Therapeutics, CHA Bundang Medical Center, CHA University School of Medicine, \\ Seongnam, Korea
}

\begin{abstract}
Objective: Although neural correlates of sub-clinical agoraphobia (AG) symptoms have been previously suggested, only a few studies evaluating structural changes of the brain have been conducted in agoraphobic patients with panic disorder (PD). We investigated and compared white matter (WM) micro-structural alterations between PD patients with $\mathrm{AG}(\mathrm{PD}+\mathrm{AG})$ and those without $\mathrm{AG}(\mathrm{PD}-\mathrm{AG})$.

Methods: Our study included 56 female PD patients, of which 25 were diagnosed with AG and 31 were diagnosed without AG. Diffusion tensor imaging was performed to investigate micro-structural changes in the WM tracts related to fronto-temporo-occipital areas (uncinate fasciculus, cingulum bundle, inferior longitudinal/fronto-occipital fasciculus, fornix column and body, and fornix/stria terminalis). All participants were subjected to the Anxiety Sensitivity Inventory-Revised (ASI-R), Beck Depression Inventory-II (BDI-II), and Albany Panic and Phobia questionnaires.

Results: The fractional anisotropy values of the right uncinate fasciculus in PD + AG were significantly lower than that of PD - AG and showed significant correlations with BDI-II and ASI-R total scores. Mean diffusivity and radial diffusivity values of the right uncinate fasciculus were significantly higher in PD + AG as compared to PD - AG. Conclusion: Our findings suggest that the uncinate fasciculus may be associated with AG symptoms in PD, possibly through demyelination. Our findings may contribute to the neurobiological evidence regarding the association between AG and WM structural changes in PD.
\end{abstract}

KEY WORDS: Panic disorder; Agoraphobia; White matter; Neuroimaging.

\section{INTRODUCTION}

Agoraphobia (AG) is defined as an anxiety disorder characterized by marked fear in situations where the person perceives their environment to be unsafe with no way to escape [1]. With the revision of the Diagnostic and Statistical Manual of Mental Disorders, fourth edition text revision (DSM-IV-TR) to the DSM-V (fifth edition), AG was

Received: January 21, 2020 / Revised: March 16, 2020

Accepted: May 6, 2020

Address for correspondence: Sang-Hyuk Lee

Department of Psychiatry, CHA Bundang Medical Center, CHA

University School of Medicine, 59 Yatap-ro, Bundang-gu,

Seongnam 13496, Korea

E-mail: leesanghyuk@yahoo.com

ORCID: https://orcid.org/0000-0001-7939-3000

Eunsoo Won

Department of Psychiatry, CHA Bundang Medical Center, $\mathrm{CHA}$

University School of Medicine, 59 Yatap-ro, Bundang-gu,

Seongnam 13496, Korea

E-mail: eunsooowon@gmail.com

ORCID: https://orcid.org/0000-0001-6825-032X re-classified as a sole psychological disorder separate from panic disorder (PD). Based on this revision, the emphasis was on AG being an independent psychological entity; hence, it was defined as a separate disorder, despite being strongly associated with PD [2]. The prevalence rate of AG without PD was reported to be $0.8 \%$, whereas that of AG with PD was 1.1\% [3]. Younger age, female sex, and comorbidity with PD and other phobias were reported to be risk factors for AG [4]. Furthermore, AG has been associated with substantial comorbidity with other DSM disorders, with higher rates of comorbidity observed in PD + AG as compared to PD - AG [3].

While providing the classical hypothesis on the neuroanatomical basis of PD, Gorman et al. [5] had emphasized the role of the fear network involving frontal and limbic regions. The uncinate fasciculus is one of the white matter (WM) tracts connecting fronto-limbic structures an association tract that connects parts of the limbic system

(c) This is an Open-Access article distributed under the terms of the Creative Commons Attribution Non-Commercial License (http://creativecommons.org/licenses/by-nc/4.0) which permits unrestricted non-commercial use, distribution, and reproduction in any medium, provided the original work is properly cited. 
such as the parahippocampus and amygdala in the temporal lobe with portions of the frontal lobe such as the orbitofrontal cortex [6]. Recent imaging studies have discovered extended areas of the fear network model (FNM), such as the sensory regions of the temporal and occipital lobes [7], to be strongly associated with PD. The visuospatial dysregulation and false threat alarm related to the temporal lobe has been well discussed by many literatures on PD $[8,9]$. Dysfunction in the processing of sensory information from fear-related stressors in the visuo-spatial system stimulates structures in the temporal region to exchange information with the autonomic nerve system and neuroendocrine system [10]. Since the occipital lobes are associated with awareness of fearful stimuli and the surrounding environment through visuo-spatial information, they are involved in transmitting sensory information to the FNM to process fear and environmental changes, which may contribute to panic attacks [11].

There are not many reports discussing the brain's structural role in AG, but structures related to the occipital and temporal lobes has been suggested to mediate AG symptoms as it shares common disease characteristics with PD, such as sensory-related fear, anxiety response, and visuospatial regulation dysfunction [12]. Anticipatory anxiety commonly observed in AG and PD could be mediated by brain activity dysfunction of the occipital lobe [13]. Hyperactivation of the temporal lobe was reported in anticipating AG-specific stimuli [14]. A recent network study on sub-clinical AG demonstrated a lower level of efficiency and clustering of the visuo-spatial-emotional network, including the amygdala, primary visual cortex, visual stream, parietal lobes, and prefrontal cortices. Hence, this lower connectivity suggests that sub-clinical AG symptoms may not organize all of this information into high-level executive function, and may react more intuitively to AG stimuli [15].

Recently, in patients with sub-clinical AG symptoms, positive correlations between symptom severity and cortical volumes of temporo-occipital areas, parts of the extended fear network, including bilateral calcarine sulci, right lingual gyrus, left superior, middle and inferior temporal gyri areas, have been reported [12]. We suggest that WM tracts associated with the extended fear network and fronto-temporo-occipital areas may underline the neural correlates of AG. Imaging techniques associated with diffusion tensor imaging (DTI) have made it possible to in- vestigate alterations in the micro-structural integrity of the WM tracts [16].

Given the above considerations, we investigated and compared the WM micro-structural alterations between PD + AG and PD - AG. First, we hypothesized that PD + AG would exhibit altered integrity in the WM tracts related to fronto-temporo-occipital areas as compared to PD - AG. Second, we hypothesized that AG symptom severity will show significant correlations with WM integrity in PD + AG.

\section{METHODS}

\section{Participants and Clinical Assessment}

We studied 56 female patients with PD who were recruited from the outpatient psychiatric clinic of $\mathrm{CHA}$ Bundang Medical Center. All participants were between 18 and 65 years of age, right-handed, and of Korean ethnicity. The medical histories of the participants were recorded through interviews and questionnaires. Diagnoses of PD + AG and PD - AG were determined by experienced psychiatrists according to the DSM-IV-TR, using the Structured Clinical Interview for DSM-IV Axis I disorders [17]. Only patients with primary diagnosis of PD were included. The exclusion criteria were as follows: (1) Primary or comorbid psychiatric diagnoses on Axis I or Axis II (based on DSM-IV-TR criteria) other than PD; (2) history of serious or unstable medical illness; (3) abnormal findings in physical examination and routine laboratory tests; (4) primary neurological illness; (5) pregnancy; and (6) any contraindications for brain magnetic resonance (MR) scanning. Fifty-four patients were consuming psychotropic medications, including selective serotonin reuptake inhibitors such as escitalopram and paroxetine (escitalopram equivalent dosage, $8.33 \pm 7.09 \mathrm{mg} /$ day), and benzodiazepines such as alprazolam and clonazepam (alprazolam equivalent dosage, $1.43 \pm 1.17 \mathrm{mg} /$ day). Brain MR scans of PD + AG and PD - AG were obtained after initiation of medication after $3.92 \pm 3.81$ and $5.87 \pm$ 5.71 days, respectively. Two PD + AG did not consume any type of psychotropic medication within the study period.

To evaluate symptom severities, the Anxiety Sensitivity Inventory-Revised (ASI-R), Albany Panic and Phobia Questionnaire (APPQ), Beck Depression Inventory-II (BDI-II), and Beck Anxiety Inventory (BAI) were conducted by all 
participants. Anxiety sensitivity levels were assessed using the Korean version of the ASI-R [18,19], which consists of fear of a respiratory symptom, fear of a cardiovascular symptom, fear of cognitive dyscontrol, and fear of a publicly observable anxiety reaction. The internal consistency coefficient of the Korean version is 0.92 and its test-retest reliability is 0.82. The APPQ was developed to assess fear of activities that may induce physical sensation in PD patients. The Korean version of the APPQ, showed good internal consistency (Cronbach's alpha $=0.95)$ and high test-retest reliability $(r=0.77)$ [20]. APPQ has three sub-scales: interoceptive avoidance, AG, and social phobia [21].

This study was approved by the CHA Bundang Medical Center Ethics Committee (no. 2011-11-164, 2018-06029, 2019-05-030). All procedures involved in this study complied with CHA Bundang Medical Center Institutional Review Board regulations, Declaration of Helsinki principles, and Good Clinical Practice principles. All participants fully understood the protocol of the research and provided their oral and written consents.

\section{MRI Acquisition}

All participants underwent MR imaging on the 3.0 T GE Signa HDxt scanner (GE Healthcare, Milwaukee, WI, USA) comprising of an eight-channel phase-array head coil at the CHA Bundang Medical Center, CHA University, and diffusion data were acquired on a 3.0 T GE Signa HDxt scanner (GE Healthcare). Diffusion-weighted images were processed using an echo planar imaging (EPI) sequence with the following parameters: repetition time (TR) of 17,000 ms, echo time (TE) of $108 \mathrm{~ms}$, field of view (FOV) of $24 \mathrm{~cm}, 144 \times 144$ matrix, $1.7 \mathrm{~mm}$ slice thickness, and voxel size of $1.67 \times 1.67 \times 1.7 \mathrm{~mm}^{3}$. A double-echo option was applied to minimize the effect of eddy current. An eight-channel coil and an array of spatial sensitivity encoding techniques (ASSET, GE Healthcare) with a sensitivity encoding (SENSE) speed-up factor of two were used to reduce the impact of EPI spatial distortions. Seventy axial slices parallel to the anterior commissure-posterior commissure (AC-PC) line covering the whole brain were acquired in 51 directions with b-value $=900 \mathrm{~s} / \mathrm{mm}^{2}$. Eight baseline scans with $b=0 \mathrm{~s} / \mathrm{mm}^{2}$ were also acquired. DTIs were approximated from the diffusion-weighted images using the least-squares method (approximate scan time = $17 \mathrm{~min})$.

\section{Image Processing}

Voxel-wise analysis of DTI metrics data was evaluated using Tract-Based Spatial Statistics (TBSS version 1.2) applied in the Oxford functional MRI of the brain (FMRIB) Software Library (FSL version 4.1, Oxford, UK; https://fsl. fmrib.ox.ac.uk/fsl/fslwiki) according to the standard procedure [22]. First, DTI preprocessing, including skull stripping using the Brain Extraction Tool (BET) and eddy current correction, were performed using the FSL. Accordingly, FA images were constructed by fitting a tensor model to the raw diffusion data [23]. The FA data of all the participants were aligned in the standard space (Montreal Neurologic Institute 152 standard) using the FMRIB's nonlinear image registration tool (FNIRT). All transformed FA images were combined and applied to the original FA map, resulting in a standard-space version of the FA map. All transformed FA images were averaged to create a mean FA image, which was thinned (skeletonized) to create a mean FA skeleton, including only the centers of the WM tracts. The skeleton was thresholded using an FA > 0.2 (TBSS default) to include only major fiber bundles. To compare the axial diffusivity (AD), radial diffusivity (RD), and mean diffusivity (MD), we used FSL of the FA images to achieve non-linear registration and skeletonization stages, as well as to estimate the projection vectors from each individual participant onto the mean FA skeleton. The non-linear warps and skeleton projection can also be applied to other images.

For the analysis of neural correlates of PD + AG vs. PD AG, mean FA skeletons were multiplied using the Johns Hopkins University (JHU) DTI-based probabilistic tractography atlas. Subsequently, the aforementioned regions of interests (ROIs) were extracted using the three-dimensional Slicer version 3.6 to create a mask (threshold: $p=$ 0.05 ) and perform voxel-wise statistical analysis [24]. The selected WM ROls included the fornix, stria terminalis, cingulum bundle, uncinate fasciculus, and inferior longitudinal/fronto-occipital fasciculus from the JHU WM atlas, based on previous studies $[12,15]$. These fronto-temporo-occipital regions were involved in the extended fear network linked with AG symptoms (Fig. 1).

The FA index is the most commonly and widely used parameter of DTI, as it detects the integrity of WM fibers [25]. FA indicates various characteristics of the axon fibers, with increased values representing either a greater number and size of the axon fibers or a decrease in the 
density of the axon fibers [26]. Previous studies have reported AD to be sensitive to the axonal pathogenesis, MD to be sensitive to necrosis and edema, and RD to be sensitive to myelination, which may correspond to the WM micro-integrity that in turn affects the functional connection of the axonal fibers [27]. Therefore, values for AD, RD, and MD could supplement FA values in order to analyze the potential underlying pathogenesis in tissue micro-structure $[26,27]$.

\section{Statistical Analysis}

To compare the sociodemographic and clinical data between PD + AG and PD - AG, the independent $t$ test

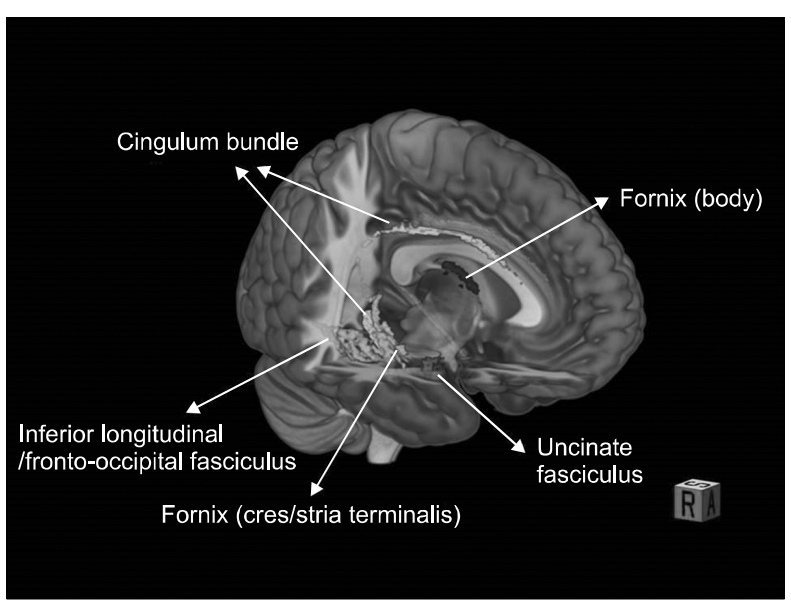

Fig. 1. 3D reconstructions of the regions of interest. was used. The independent $t$ test was applied with multiple test correction using post-hoc Benjamini-Hochberg false discovery rate to compare the FA values between PD + AG and PD - AG. To ensure the results were not confounded by other variables, such as age and intracranial volume, analysis of covariance (ANCOVA) was performed. Spearman correlation was used in each PD group for non-hypothetical exploratory analysis.

All statistical analyses were performed using the Statistical Package for the Social Sciences (IBM SPSS statistics 25.0 software; IBM Co., Armonk, NY, USA). For all tests, $p<$ 0.05 was considered statistically significant.

\section{RESULTS}

\section{Sociodemographic Characteristics}

Table 1 summarizes the sociodemographic and clinical characteristics of PD + AG and PD - AG There were no statistically significant differences between the two diagnostic groups, in terms of age at the time of scan, years of education, and intracranial volume (ICV). APPQ, BAI, BDI-II, and ASI-R total scores, along with APPQ sub-scale scores, were significantly higher in PD + AG as compared to PD - AG.

\section{Group Differences in FA, AD, MD, and RD Values}

In the comparison between $\mathrm{PD}+\mathrm{AG}$ and $\mathrm{PD}-\mathrm{AG}$, significant difference in the $\mathrm{FA}$ values of the uncinate fas-

Table 1. Demographic and clinical characteristics of the panic disorder participants

\begin{tabular}{|c|c|c|c|c|}
\hline \multirow{2}{*}{ Variable } & \multirow{2}{*}{$P D-A G(n=31)$} & \multirow{2}{*}{$\mathrm{PD}+\mathrm{AG}(\mathrm{n}=25)$} & \multicolumn{2}{|c|}{ Statistics } \\
\hline & & & $t$ & $p$ value \\
\hline Age $(y r)$ & $35.52 \pm 10.83$ & $38.44 \pm 10.53$ & -1.02 & 0.314 \\
\hline Education (yr) & $13.32 \pm 2.99$ & $13.08 \pm 3.17$ & 0.30 & 0.766 \\
\hline Intracranial volumes $\left(\mathrm{mm}^{3}\right)$ & $1,400.27 \pm 119.08$ & $1,361.63 \pm 87.28$ & 1.14 & 0.262 \\
\hline BDI-II, total score & $15.06 \pm 7.58$ & $22.96 \pm 10.75$ & -3.19 & 0.002 \\
\hline BAI, total score & $23.10 \pm 11.52$ & $36.17 \pm 13.57$ & -3.86 & 0.000 \\
\hline ASI-R, total score & $44.97 \pm 19.20$ & $68.46 \pm 29.22$ & -3.38 & 0.002 \\
\hline APPQ, total score & $31.94 \pm 21.38$ & $105.58 \pm 35.03$ & -9.08 & 0.000 \\
\hline Agoraphobia & $10.77 \pm 7.11$ & $44.16 \pm 13.06$ & -11.48 & 0.000 \\
\hline Social phobia & $10.00 \pm 8.85$ & $30.40 \pm 17.83$ & -5.23 & 0.000 \\
\hline Interoception & $11.16 \pm 9.98$ & $31.29 \pm 12.37$ & -6.68 & 0.000 \\
\hline SSRI escitalopram equivalent dosage (mg/day) $)^{a}$ & $7.5 \pm 7.33$ & $9.40 \pm 6.78$ & -0.98 & 0.330 \\
\hline Benzodiazepine equivalent dosage $(\mathrm{mg} / \text { day })^{b}$ & $1.46 \pm 1.45$ & $1.39 \pm 0.73$ & -0.21 & 0.830 \\
\hline
\end{tabular}

Values are presented as mean \pm standard deviation.

PD, panic disorder; AG, agoraphobia; BDI-II, Beck Depression Inventory-II; BAI, Beck Anxiety Inventory; ASI-R, Anxiety Sensitivity Index-Revised; APPQ, Albany Panic and Phobia Questionnaire; SSRI, selective serotonin reuptake inhibitor.

${ }^{a}$ The approximate equivalent oral doses to $10 \mathrm{mg}$ escitalopram are given. ${ }^{\mathrm{b}}$ The approximate equivalent oral doses to $1 \mathrm{mg}$ lorazepam are given. 
ciculus was observed (Table 2). PD + AG showed significantly lower $\mathrm{FA}$ values as compared to PD $-\mathrm{AG}(t=$ 4.21, Benjamini-Hochberg False Discovery Rate [FDR]corrected $p=0.021)$. The MD and RD values of PD + AG were significantly higher than PD - AG, while no significant difference was observed in the $\mathrm{AD}$ values between the two groups (MD, corrected $p=0.041 ; \mathrm{RD}$, corrected $p=0.034)$. No significant difference in the other ROls was observed between PD - AG and PD + AG (Table 2). Moreover, no significant correlation was shown between the FA values of the right uncinate fasciculus and age, years of education, duration of medication, medicine dosage, and ICV. Furthermore, the difference in FA values of the uncinate fasciculus remained significant when
ANCOVA was performed with the aforementioned covariates. There was no significant interaction effect between the ROIs according to laterality. That is, there was no significant difference between the FA values of the right and left uncinate fasciculus. In addition, there was no interaction effect between laterality and groups

\section{Exploratory Correlation Analysis between FA Values of the Uncinate Fasciculus and Clinical Symptom Severity in Patients with PD}

In PD + AG, the FA values of the uncinate fasciculus showed significant correlations with BDI-II and ASI-R total scores, as well as the ASI-R cognitive sub-scale scores $(r=0.476, p=0.02 ; r=0.405, p=0.049$; and $r=0.413$,

Table 2. Comparison of FA values of ROls between PD + AG and PD - AG

\begin{tabular}{|c|c|c|c|c|}
\hline \multirow{2}{*}{ Regions of interest } & \multirow{2}{*}{$P D-A G(n=31)$} & \multirow{2}{*}{$P D+A G(n=25)$} & \multicolumn{2}{|c|}{ Statistics } \\
\hline & & & $t$ & $p$ value $^{\mathrm{a}}$ \\
\hline Fornix (column and body) & $0.66 \pm 0.13$ & $0.62 \pm 0.11$ & 0.981 & 0.328 \\
\hline \multicolumn{5}{|l|}{ Left } \\
\hline $\begin{array}{l}\text { Inferior longitudinal/ } \\
\text { fronto-occipital fasciculus }\end{array}$ & $0.63 \pm 0.03$ & $0.63 \pm 0.04$ & 0.381 & 0.705 \\
\hline Fornix (cres/stria terminals) & $0.65 \pm 0.03$ & $0.65 \pm 0.04$ & 0.809 & 0.374 \\
\hline Uncinate fasciculus & $0.63 \pm 0.04$ & $0.62 \pm 0.06$ & 3.481 & 0.069 \\
\hline Cingulum bundle & $0.73 \pm 0.05$ & $0.73 \pm 0.04$ & 0.388 & 0.669 \\
\hline \multicolumn{5}{|l|}{ Right } \\
\hline $\begin{array}{l}\text { Inferior longitudinal/ } \\
\text { fronto-occipital fasciculus }\end{array}$ & $0.67 \pm 0.03$ & $0.66 \pm 0.03$ & 1.158 & 0.252 \\
\hline Fornix (cres/stria terminalis) & $0.71 \pm 0.03$ & $0.70 \pm 0.04$ & 0.001 & 0.97 \\
\hline Uncinate fasciculus & $0.65 \pm 0.04$ & $0.62 \pm 0.06$ & 4.206 & 0.021 \\
\hline Cingulum bundle & $0.77 \pm 0.04$ & $0.77 \pm 0.04$ & 0.322 & 0.541 \\
\hline
\end{tabular}

Values are presented as mean \pm standard deviation.

FA, fractional anisotropy; ROI, region of interest; PD, panic disorder; AG, agoraphobia.

${ }^{\mathrm{a}} p$ values were corrected for multiple comparisons using a Benjamini-Hochberg false discovery rate correction method.


Fig. 2. Exploratory correlation analysis between FA value of right uncinate fasciculus and clinical symptom severity in PD + AG. PD, panic disorder; AG, agoraphobia; ASI-R, Anxiety Sensitivity Inventory-Revised; BDI-II, Beck Depression Inventory-II. 


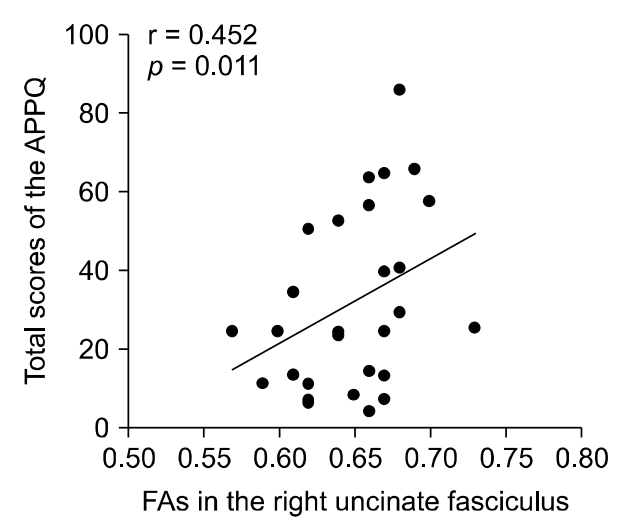

$p=0.04$, respectively). In PD-AG, FA values of the uncinate fasciculus significantly correlated with the total APPQ and APPQ interoception sub-scale scores $(r=$ $0.452, p=0.011 ; r=0.389, p=0.031$, respectively). Nevertheless, after multiple comparisons with Bonferroni correction, the $p$ values did not survive (Figs. 2, 3).

\section{Association Analysis Using Multiple Linear Regression}

In $\mathrm{PD}+\mathrm{AG}$, the multiple linear regression analysis showed FA value of the uncinate fasciculus to be significantly associated with ASI-Cog $(p=0.026)$, ASI-SUM $(p=0.018)$, and BDI-SUM ( $p=0.014)$, with age, ICV, education, and medication effect included as covariates in the analysis.

In PD - AG, the multiple linear regression analysis showed FA value of the uncinate fasciculus to be significantly associated with APPQ sum $(p=0.039)$, but not with APPQ interoception, with age, ICV, education, and medication effect included as covariates in the analysis.

\section{DISCUSSION}

To the best of our knowledge, this is the first study to examine and compare WM structural changes between PD + AG and PD - AG. The present study observed that the FA values of the uncinate fasciculus in $\mathrm{PD}+\mathrm{AG}$ were significantly lower than that of PD - AG. Furthermore, FA values of the uncinate fasciculus in each group showed positive correlations with symptom severities.

The results of the present study indicate decreased integrity of the uncinate fasciculus in PD + AG as compared to PD - AG. The uncinate fasciculus connects the prefrontal lobe and amygdala, which are central in controlling amygdala-related fear responses [28]. Generally, negative correlations have been observed between structural integrity and anxiety trait levels (i.e., higher FA values predicted lower anxiety levels) [29]. Furthermore, the uncinate fasciculus has also been associated with executive functions, such as associative and episodic memory functions and social-emotion functions [30].

High AG-related anxiety is closely associated with spatial and visual processing and activation of autobiographical memory to interpret the surrounding environment. Commonly, patients describe this arousal as more distressing and anxiety inducing in daily life than confronting and staying in the agoraphobic situation [31]. Functionally, impaired integrity of the uncinate fasciculus could contribute to the disconnectivity between the prefrontal cortex and amygdala. This may lead to the dysfunction in inhibitory control of the prefrontal cortex on the amygdala, and influence amygdala-related fear responses. Episodic memory functions and social-emotion functions may also be impaired, affecting the anticipatory anxiety related to AG and misinterpreting the surrounding environment to be safe or not from previous memory. Impaired uncinate fasciculus-related executive functions could result in prominent susceptibility to phobic situations and greater risk for developing phobic symptoms, which may contribute to the development of AG symptoms.

Similar fronto-temporal structural hypo-connectivity has also been observed in patients with social anxiety disorder and generalized anxiety disorder, which indicates an association between uncinate fasciculus abnormalities and emotional regulation deficits $[32,33]$. Decreased FA values of WM around the frontal lobe were observed in patients with PD, which indicated impaired micro-connectivity of the frontal WM in PD [34]. Furthermore, anti- 
depressant therapy was shown to increase micro-integrity of the fronto-occipital fasciculus and uncinate fasciculus in PD [35]. Therefore, phobic and anxiety symptoms of AG could be related to impaired micro-connectivity of the uncinate fasciculus, which connects the frontal lobe structures including the orbitofrontal cortex and anterior cingulate cortex, and the anterior temporal lobe, including portions of the amygdala and hippocampus, which are critical structures in fear and memory modulation [36].

The exact role of the right and left uncinate fasciculus is still unknown, but one may speculate from the results of previous studies which have focused on hemispheric lateralization in psychiatric disorders [37]. Depression has often been associated with left brain damage [38], and panic anxiety has been associated with right cortical disturbance [39]. In rodents, Andersen et al. found increased serotonin levels in the right, but not the left amygdala in relation to anxiety, which also suggested anxiety to be associated with right hemispheric function [40].

Numerous case reports have reported associations between right sided brain lesions such as tumors and anxiety $[41,42]$, and certain studies have suggested left cortical lesions to be associated with anxiety combined with depression, and right hemisphere lesions to be associated with anxiety alone [43].

Furthermore, our results showing decreased FA values of the right uncinate fasciculus are compatible with previous studies reporting acute and chronic stress to result in greater involvement of the right hemisphere [44] and the effect of life stress on reduced FA of right uncinate fasciculus, and higher level of anxiety symptoms [45]. Such results of previous studies suggested anxiety and stress to be associated more with right hemispheric function compared to the left, and this may be why our results showed only significant results in the right uncinate fasciculus and not the left uncinate fasciculus.

Second, the degree of directionality of diffusion and the diffusivity in a tissue can be measured by FA and MD, respectively. Extending the knowledge from the evaluation of FA and MD, recent studies showed AD (presumably linked with directional diffusivity along the axon) and RD (presumably linked with diffusivity perpendicular to the axon) underpin the biological alterations such as axonal and myelin changes $[46,47]$. Our findings of increases in both $\mathrm{RD}$ and $\mathrm{MD}$ values of the right uncinate fasciculus suggest a possible contribution of demyelination to the AG-related reduction in the right uncinate fasciculus integrity in the PD group. Impairment of myelin could contribute to frontal lobe inhibition and emotional dysregulation related to AG. Furthermore, this trend of FA and RD values was observed in many other DTI studies [48,49].

Third, significant correlations were observed between the FA values of the uncinate fasciculus and the BDI-II, ASI-R total, and ASI-R cognitive sub-scale scores in PD + AG. Correlations between FA values of the uncinate fasciculus and APPQ scores were observed only in PD - AG in our study. Although there was no significant difference in the prevalence of major depressive disorder between patients with (15.3\%) and without AG (12.7\%) in PD [50], agoraphobia is reported to be one of the risk factors for major depressive disorder [51]. Also, the importance of anxiety sensitivity has been emphasized as a predictor of agoraphobia symptoms $[52,53]$. We considered our results to be similar to the results of previous studies reporting the presence of agoraphobia to be associated with depression and anxiety vulnerability, as BDI-II assesses depression symptoms and ASI-R assesses anxiety sensitivity. Only APPQ scores were associated with FA values in PD AG, with the APPQ assessing fear of activities that may elicit physical sensation in panic patients [21].

However, the correlations between FA values of the uncinate fasciculus and symptom severities were positive rather than negative. A little research is done to explain such a finding but we may speculate that decreased integrity of the uncinate fasciculus in PD + AG may influence $\gamma$-aminobutyric acid GABAnergic inhibitory transmission of the uncinate fasciculus [54]. Dysfunction of GABAnergic inhibitory transmission can influence the local axonal environment and mediate myelin dysfunction and overactivation, which may lead to increased FA values [55]. Patients with higher symptom severities may have further dysfunction in GABAnergic inhibitory transmission, hence present paradoxically increased FA values. Such paradoxic increases in FA values, due to possible disruption to the inhibitory GABAnergic system, have been reported in previous studies [56-58]. Further studies are needed to explain speculative assumption.

This study had several limitations. First, only females were included in this study. Although we purposely included only female subjects in our study due to the higher prevalence of AG in females compared to males, the fact 
that all subjects were females may be considered as a limitation in the context of generalizing our findings [59]. Second, we relied on a relatively small sample size. While our sample size was similar to that of recent neuroimaging studies, future studies including a larger sample size would be helpful in demonstrating confident results [60,61]. Third, 55 patients were consuming antidepressants during the study, and several studies have found a relationship between structural changes of the brain and antidepressant treatment [62]. However, our results remained statistically significant even after correcting for duration of medication as a covariate.

In conclusion, we provide evidence for decreased integrity of the uncinate fasciculus in PD + AG as compared to PD - AG. Additionally, our results suggest an association between uncinate fasciculus integrity and depression and anxiety vulnerability in PD + AG. Our study provides further neurobiological evidence on structural brain changes in AG.

\section{Acknowledgments}

This research was supported by the National Research Foundation of Korea (NRF) grants funded by the Ministry of Science and ICT, Republic of Korea (Grant No. NRF-2011-0023359, NRF-2018R1D1A1B07046978, and NRF-2019M3C7A1032262). The funding sources had no further role in the study design; collection, analysis, and interpretation of data; writing of the paper; and decision to submit the paper for publication. We are thankful to Iseul An for her technical support.

\section{Conflicts of Interest}

No potential conflict of interest relevant to this article was reported.

\section{- Author Contributions}

Conceptualization: Sung Eun Kim and Sang-Hyuk Lee. Data acquisition: Sung Eun Kim. Formal analysis: Sung Eun Kim. Funding: Sang-Hyuk Lee. Supervision: Minji Bang, Eunsoo Won, and Sang-Hyuk Lee. Writing - original draft: Sung Eun Kim and Sang-Hyuk Lee. Writing - review \& editing: Sung Eun Kim, Eunsoo Won, and Sang-Hyuk Lee.

\section{ORCID}

Sung Eun Kim

https://orcid.org/0000-0002-0983-9932

Minji Bang
Eunsoo Won

https://orcid.org/0000-0001-6825-032X

Sang-Hyuk Lee

https://orcid.org/0000-0001-7939-3000

\section{REFERENCES}

1. Asmundson GJ, Taylor S, Smits JA. Panic disorder and agoraphobia: an overview and commentary on DSM-5 changes. Depress Anxiety 2014;31:480-486.

2. Wittchen HU, Gloster AT, Beesdo-Baum K, Fava GA, Craske MG. Agoraphobia: a review of the diagnostic classificatory position and criteria. Depress Anxiety 2010;27:113-133.

3. Kessler RC, Chiu WT, Jin R, Ruscio AM, Shear K, Walters EE. The epidemiology of panic attacks, panic disorder, and agoraphobia in the National Comorbidity Survey Replication. Arch Gen Psychiatry 2006;63:415-424.

4. Bracha HS, Lenze SM, Shelton J. Primary agoraphobia as a specific phobia. Br J Psychiatry 2006;189:470; author reply 471.

5. Gorman JM, Kent JM, Sullivan GM, Coplan JD. Neuroanatomical hypothesis of panic disorder, revised. Am J Psychiatry 2000; 157:493-505.

6. Kier EL, Staib LH, Davis LM, Bronen RA. MR imaging of the temporal stem: anatomic dissection tractography of the uncinate fasciculus, inferior occipitofrontal fasciculus, and Meyer's loop of the optic radiation. AJNR Am J Neuroradiol 2004;25:677-691.

7. Lai $\mathrm{CH}$. Fear network model in panic disorder: the past and the future. Psychiatry Investig 2019;16:16-26.

8. Pillay SS, Gruber SA, Rogowska J, Simpson N, Yurgelun-Todd DA. $f M R I$ of fearful facial affect recognition in panic disorder: the cingulate gyrus-amygdala connection. I Affect Disord 2006;94:173-181.

9. Windmann S. Panic disorder from a monistic perspective: integrating neurobiological and psychological approaches. J Anxiety Disord 1998;12:485-507.

10. Hermans EJ, van Marle HJ, Ossewaarde L, Henckens MJ, Qin $\mathrm{S}$, van Kesteren MT, et al. Stress-related noradrenergic activity prompts large-scale neural network reconfiguration. Science 2011;334:1151-1153.

11. van de Riet WA, Grezes J, de Gelder B. Specific and common brain regions involved in the perception of faces and bodies and the representation of their emotional expressions. Soc Neurosci 2009;4:101-120.

12. Besteher B, Squarcina L, Spalthoff R, Bellani M, Gaser C, Nenadić I, et al. Subclinical agoraphobia symptoms and regional brain volumes in non-clinical subjects: between compensation and resilience? Front Psychiatry 2018;9:541.

13. Kumari V, ffytche $\mathrm{DH}$, Das $\mathrm{M}$, Wilson GD, Goswami $\mathrm{S}$, Sharma T. Neuroticism and brain responses to anticipatory fear. Behav Neurosci 2007; 121:643-652.

14. Wittmann A, Schlagenhauf F, Guhn A, Lueken U, Gaehlsdorf C, Stoy M, et al. Anticipating agoraphobic situations: the neural correlates of panic disorder with agoraphobia. Psychol Med 2014:44:2385-2396. 
15. Indovina I, Conti A, Lacquaniti F, Staab JP, Passamonti L, Toschi N. Lower functional connectivity in vestibular-limbic networks in individuals with subclinical agoraphobia. Front Neurol 2019;10:874.

16. Won E, Kang J, Choi S, Kim A, Han KM, Yoon HK, et al. The association between substance $P$ and white matter integrity in medication-naive patients with major depressive disorder. Sci Rep 2017;7:9707.

17. Hahn OS, Ahn JH, Song SH, Cho MJ, Kim JK, Bae JN, et al. Development of Korean version of structured clinical interview schedule for DSM-IV axis I disorder: interrater reliability. I Korean Neuropsychiatr Assoc 2000;39:362-372.

18. Lim YJ, Yu BH, Kim JH. Korean Anxiety Sensitivity IndexRevised: its factor structure, reliability, and validity in clinical and nonclinical samples. Depress Anxiety 2007;24:331-341.

19. Taylor S, Cox BJ. An expanded anxiety sensitivity index: evidence for a hierarchic structure in a clinical sample. J Anxiety Disord 1998; 12:463-483.

20. Kim JH, Yang JC, Kim JB, Lim KY, Lee SY, Yu BH. A validation study of Korean Albany Panic and Phobia Questionnaire (APPQ). J Korean Neuropsychiatr Assoc 2004;43:329-336.

21. Brown TA, White KS, Barlow DH. A psychometric reanalysis of the Albany Panic and Phobia Questionnaire. Behav Res Ther 2005;43:337-355.

22. Smith SM, Jenkinson $M$, Johansen-Berg $H$, Rueckert D, Nichols TE, Mackay CE, et al. Tract-based spatial statistics: voxe/wise analysis of multi-subject diffusion data. Neuroimage 2006; 31:1487-1505.

23. Smith SM. Fast robust automated brain extraction. Human Brain Mapp 2002;17:143-155.

24. Pieper S, Halle M, Kikinis R. 3D slicer. In: 2004 2nd IEEE International Symposium on Biomedical Imaging: Nano to Macro (IEEE Cat No. 04EX821); Apr 18, 2004; Arlington (VA):IEEE;2004. p. 632-635.

25. Assaf Y, Pasternak O. Diffusion tensor imaging (DTI)-based white matter mapping in brain research: a review. J Mol Neurosci 2008;34:51-61.

26. Feldman HM, Yeatman JD, Lee ES, Barde LH, Gaman-Bean S. Diffusion tensor imaging: a review for pediatric researchers and clinicians. J Dev Behav Pediatr 2010;31:346-356.

27. Alexander AL, Hurley SA, Samsonov AA, Adluru N, Hosseinbor AP, Mossahebi P, et al. Characterization of cerebral white matter properties using quantitative magnetic resonance imaging stains. Brain Connect 2011;1:423-446.

28. Folloni D, Sallet J, Khrapitchev AA, Sibson N, Verhagen L, Mars RB. Dichotomous organization of amygdala/temporal-prefrontal bundles in both humans and monkeys. Elife 2019;8:e47175.

29. Kim MJ, Whalen PJ. The structural integrity of an amygdala-prefrontal pathway predicts trait anxiety. I Neurosci 2009; 29:11614-11618.

30. Von Der Heide RJ, Skipper LM, Klobusicky E, Olson IR. Dissecting the uncinate fasciculus: disorders, controversies and a hypothesis. Brain 2013;136(Pt 6):1692-1707.

31. Helbig-Lang S, Lang T, Petermann F, Hoyer J. Anticipatory anxiety as a function of panic attacks and panic-related self-efficacy: an ambulatory assessment study in panic disorder. Behav Cogn Psychother 2012;40:590-604.

32. Baur $\mathrm{V}$, Brühl AB, Herwig $U$, Eberle T, Rufer M, Delsignore A, et al. Evidence of frontotemporal structural hypoconnectivity in social anxiety disorder: a quantitative fiber tractography study. Hum Brain Mapp 2013;34:437-446.

33. Tromp DP, Grupe DW, Oathes DJ, McFarlin DR, Hernandez PJ, Kral TR, et al. Reduced structural connectivity of a major frontolimbic pathway in generalized anxiety disorder. Arch Gen Psychiatry 2012;69:925-934.

34. Kim B, Kim JH, Kim MK, Lee KS, Kim Y, Choi TK, et al. Frontal white matter alterations in short-term medicated panic disorder patients without comorbid conditions: a diffusion tensor imaging study. PLoS One 2014;9:e95279.

35. Lai $\mathrm{CH}, \mathrm{Wu} \mathrm{YT}, \mathrm{Yu} \mathrm{PL}$, Yuan W. Improvements in white matter micro-structural integrity of right uncinate fasciculus and left fronto-occipital fasciculus of remitted first-episode medication-naïve panic disorder patients. J Affect Disord 2013; 150:330-336.

36. Zhang A, Leow A, Ajilore O, Lamar M, Yang S, Joseph J, et al. Quantitative tract-specific measures of uncinate and cingulum in major depression using diffusion tensor imaging. Neuropsychopharmacology 2012;37:959-967.

37. Cummings JL. Neuropsychiatric manifestations of right hemisphere lesions. Brain Lang 1997;57:22-37.

38. Vataja R, Pohjasvaara T, Leppävuori A, Mäntylä R, Aronen HJ, Salonen $\mathrm{O}$, et al. Magnetic resonance imaging correlates of depression after ischemic stroke. Arch Gen Psychiatry 2001; 58:925-931.

39. Wiedemann G, Pauli P, Dengler W, Lutzenberger W, Birbaumer N, Buchkremer G. Frontal brain asymmetry as a biological substrate of emotions in patients with panic disorders. Arch Gen Psychiatry 1999;56:78-84.

40. Andersen SL, Teicher MH. Serotonin laterality in amygdala predicts performance in the elevated plus maze in rats. Neuroreport 1999;10:3497-3500.

41. Kellner M, Hirschmann M, Wiedemann K. Panic attacks caused by temporal tumors: an exemplary new case and a review. Depress Anxiety 1996;4:243-245.

42. Mainio A, Hakko H, Niemelä A, Tuurinkoski T, Koivukangas J, Räsänen P. The effect of brain tumour laterality on anxiety levels among neurosurgical patients. I Neurol Neurosurg Psychiatry 2003;74:1278-1282.

43. Castillo CS, Starkstein SE, Fedoroff JP, Price TR, Robinson RG. Generalized anxiety disorder after stroke. J Nerv Ment Dis 1993;181:100-106

44. Ocklenburg S, Korte SM, Peterburs J, Wolf OT, Güntürkün O. Stress and laterality - the comparative perspective. Physiol Behav 2016;164(Pt A):321-329.

45. Ho TC, King LS, Leong JK, Colich NL, Humphreys KL, Ordaz 
SJ, et al. Effects of sensitivity to life stress on uncinate fasciculus segments in early adolescence. Soc Cogn Affect Neurosci 2017;12:1460-1469.

46. Song SK, Sun SW, Ramsbottom MJ, Chang C, Russell J, Cross AH. Dysmyelination revealed through MRI as increased radial (but unchanged axial) diffusion of water. Neuroimage 2002;17:1429-1436.

47. Kinoshita Y, Ohnishi A, Kohshi K, Yokota A. Apparent diffusion coefficient on rat brain and nerves intoxicated with methy/mercury. Environ Res 1999;80:348-354.

48. Guo Y, Gao F, Liu Y, Guo H, Yu W, Chen Z, et al. White matter microstructure alterations in patients with spinal cord injury assessed by diffusion tensor imaging. Front Hum Neurosci 2019;13:11.

49. Madden DJ, Bennett IJ, Burzynska A, Potter GG, Chen NK, Song AW. Diffusion tensor imaging of cerebral white matter integrity in cognitive aging. Biochim Biophys Acta 2012; 1822:386-400.

50. Maser JD. Comorbidity of mood and anxiety disorders. Washington:American Psychiatric Press; 1990.

51. Bittner A, Goodwin RD, Wittchen HU, Beesdo K, Höfler M, Lieb R. What characteristics of primary anxiety disorders predict subsequent major depressive disorder? J Clin Psychiatry 2004;65:618-626, quiz 730 .

52. Hayward C, Wilson KA. Anxiety sensitivity: a missing piece to the agoraphobia-without-panic puzzle. Behav Modif 2007; 31:162-173.

53. Jurin T, Biglbauer S. Anxiety sensitivity as a predictor of panic disorder symptoms: a prospective 3-year study. Anxiety Stress Coping 2018;31:365-374.

54. Aroniadou-Anderjaska V, Qashu F, Braga MF. Mechanisms regulating GABAergic inhibitory transmission in the basolateral amygdala: implications for epilepsy and anxiety disorders. Amino Acids 2007;32:305-315.

55. Hamilton NB, Clarke LE, Arancibia-Carcamo IL, Kougioumtzidou E, Matthey M, Káradóttir R, et al. Endogenous GABA controls oligodendrocyte lineage cell number, myelination, and CNS internode length. Glia 2017;65:309-321.

56. Kim MK, Kim B, Lee KS, Kim CM, Bang SY, Choi TK, et al. White-matter connectivity related to paliperidone treatment response in patients with schizophrenia. J Psychopharmacol 2016;30:294-302.

57. Shergill SS, Kanaan RA, Chitnis XA, O'Daly O, Jones DK, Frangou $\mathrm{S}$, et al. A diffusion tensor imaging study of fasciculi in schizophrenia. Am J Psychiatry 2007;164:467-473.

58. Koch K, Wagner G, Dahnke R, Schachtzabel C, Schultz C, Roebel $\mathrm{M}$, et al. Disrupted white matter integrity of corticopontine-cerebellar circuitry in schizophrenia. Eur Arch Psychiatry Clin Neurosci 2010;260:419-426.

59. Goodwin RD, Faravelli C, Rosi S, Cosci F, Truglia E, de Graaf $\mathrm{R}$, et al. The epidemiology of panic disorder and agoraphobia in Europe. Eur Neuropsychopharmacol 2005; 15:435-443.

60. Kang JM, Joo SW, Son YD, Kim H, Ko KP, Lee JS, et al. Low white-matter integrity between the left thalamus and inferior frontal gyrus in patients with insomnia disorder. J Psychiatry Neurosci 2018;43:366-374.

61. Yang C, Zhang Y, Lu M, Ren J, Li Z. White matter structural brain connectivity of young healthy individuals with high trait anxiety. Front Neurol 2020;10:1421.

62. Smith R, Chen K, Baxter L, Fort C, Lane RD. Antidepressant effects of sertraline associated with volume increases in dorsolateral prefrontal cortex. J Affect Disord 2013;146:414-419. 